\title{
APROXIMACIÓN AL CONCEPTO DE CONJUNTOS DE SABERES POPULARES GANADEROS EN URUGUAY
}

\author{
Maximiliano Piedracueva Coronel ${ }^{1}$
}

\begin{abstract}
RESUMEN
La extensión rural y agraria ha rondado siempre el debate en cuanto a cuál es la mejor forma de compartir, difundir, extender o construir un conocimiento validado que permita una mejora en las condiciones de vida de la población. En el presente trabajo se aborda el análisis de las prácticas de extensión desde una mirada epistemológica. A partir de una revisión teórica y de documentos con relatos empíricos se propone una matriz de análisis en base al concepto de conjunto de saberes, con ello se intenta romper con la dicotomía de saber popular-saber científico. El objetivo es identificar de forma aproximada algunos criterios de validación en el conjunto de saberes populares de la ganadería familiar del norte del Uruguay.
\end{abstract}

Palabras clave: conjunto de saberes, extensión rural, epistemología.

\section{ABORDAGEM DO CONCEITO DE UM CONJUNTO DE CONHECIMENTOS POPULARES DE PECUÁRIA NO URUGUAI}

\section{RESUMO}

A extensão rural e agrária sempre assombrou o debate sobre qual a melhor forma de compartilhar, disseminar, ampliar ou construir um conhecimento validado que permita uma melhoria nas condições de vida da população. No presente trabalho, a análise das práticas de extensão é abordada do ponto de vista epistemológico. A partir de uma revisão teórica e de documentos com histórias empíricas, propõe-se uma matriz de análise a partir do conceito de conjunto de saberes, com isso busca-se romper com a dicotomia saber popular-saber científico. O objetivo é identificar, grosso modo, alguns critérios de validação no conjunto de conhecimentos populares sobre a agricultura familiar no norte do Uruguai.

Palavras-chave: conjunto de saberes, extensão rural, epistemologia.

\section{INTRODUCCIÓN}

La extensión rural y agraria, en sus diferentes enfoques, ha rondado siempre el debate en cuanto a cuál es la mejor forma (sea técnica, ética o ideológica) de compartir, difundir, extender o construir un conocimiento validado que permita una

\footnotetext{
${ }^{1}$ Magíster en Ciencias Agrarias por la Facultad de Agronomía de la Universidad de la República (Udelar). Docente del Departamento de Ciencias Sociales del Centro Universitario Regional Litoral Norte, UdelaR; y del Servicio Central de Extensión y Actividades en el Medio, Udelar. E-mail: maxipc85@gmail.com
} 
mejora en las condiciones de vida de la población. Desde la Agronomía Social planteada por Alexis Chayanov (1918, citado por Sánchez de la Puerta, 1996), pasando por la teoría funcionalista de Difusión de Innovaciones de Rogers (1962), la Educación popular de Paulo Freire (1983) y hasta la ecología de saberes de De Sousa Santos (2010), ha existido siempre un eje de debate: el rol del conocimiento (particularmente el científico) frente a las transformaciones de la sociedad.

En el presente trabajo se aborda este debate desde un análisis epistemológico de las prácticas de extensión. El objetivo es ahondar en la reflexión y el análisis de la dimensión epistémica de la extensión agraria, y en concreto agregar algunos elementos de discusión epistemológica sobre el saber popular agrario en la ganadería familiar del Uruguay. Se entiende que uno de los elementos centrales, y no tan estudiado, refiere a los mecanismos de validación del saber-conocimiento desde la lógica de las familias ganaderas. Estos mecanismos son fundamentales para poder emprender un posible camino de diálogo e intercambio de distintos conjuntos de saberes.

El objetivo trazado se aborda a través de una revisión bibliográfica de la literatura vinculada a la extensión crítica y a las epistemologías críticas, así como a partir de trabajos empíricos realizados en el Uruguay sobre la ganadería familiar y sus vínculos con las tecnologías agropecuarias. La selección de los trabajos teóricos es arbitraria dado el objetivo planteado y, por tanto, se han seleccionado aquellos textos que habilitan una reconstrucción conceptual del conocimiento como construcción social: filosofía de la ciencia, sociología de la ciencia, epistemologías críticas, Educación popular, Investigación Acción Participación y epistemologías del sur. Los trabajos empíricos han sido seleccionados mediante la revisión de publicaciones específicas en el Uruguay: revistas del Instituto Nacional de Investigación Agropecuaria (INIA), revistas del Instituto Plan Agropecuario (IPA), revistas Agrociencia de la Facultad de Agronomía; a partir de artículos o documentos ubicados en esas publicaciones se ha accedido a la lectura de otros documentos conexos con otras fuentes ${ }^{2}$.

El artículo se inicia con una reconstrucción discursiva sobre el conocimiento como construcción social a partir de los aportes de la sociología de la ciencia y las epistemologías críticas. Seguidamente se sitúa el análisis en la producción ganadera familiar del Uruguay presentando algunos de los principales resultados de estudios empíricos e informes de trabajo que han abordado el vínculo entre saber técnico y tecnologías en la ganadería familiar uruguaya. El siguiente apartado incorpora una lectura del proceso de diálogo entre los saberes científicos y populares a través de la ecología de saberes, principalmente retomando la categoría de traducción intercultural como opción mediadora en el diálogo. El apartado cuarto presenta y pone en reflexión el concepto de conjuntos de saberes como categoría analítica que permite profundizar el análisis y romper con la dicotomía de saber científico-saber popular; en este espacio se presentan algunos de los resultados encontrados en la revisión respecto a cuáles pueden ser los criterios de validación del conocimiento en el conjunto de saberes populares de la ganadería. El quinto apartado presenta algunas consideraciones finales donde se resalta la necesidad de continuar profundizando en el análisis de la relación social de producción y validación del conocimiento bajo una mirada de conjunto de saberes.

\footnotetext{
${ }^{2}$ El objetivo no es realizar un análisis del discurso científico sobre la temática sino recoger los principales abordajes que ha tenido en el Uruguay. Por tal motivo, la selección no es una muestra representativa de la producción existente en el país.
} 


\title{
1.1 LA CONSTRUCCIÓN DE CONOCIMIENTO
}

Desde que surge la ciencia moderna como institución social en el mundo occidental (Merton, 1977) se han sucedido debates sobre la legitimidad y la potencialidad de los conocimientos adquiridos, generados o construidos, según sea el enfoque. Muchos han sido los aportes dentro de este debate de algunas disciplinas tales como la historia de la ciencia, la sociología de la ciencia, la epistemología, la gnoseología, la filosofía de la ciencia, la extensión y la metodología (tanto de intervención como de investigación). El debate que aquí nos convoca es transversal a los ejes de discusión que en ellas se han tratado. ¿Qué conocimiento? ¿Para qué? Y entonces ¿cómo?, son algunos de los ejes que guían la discusión del saber popular, tradicional y metafísico con el conocimiento técnico y científico.

En este trabajo se diferencia analíticamente el conocimiento del saber, y por otra parte se hablará también de un conjunto de saberes. El saber es entendido como conocimiento tácito (Polanyi, 1967) mientras que el conocimiento es referido aquí como un saber abstracto, trasmisible, y que permite que sea utilizado en una acción (MANRIQUE, 2008). El conocimiento, por tanto, refiere a un componente de abstracción; por ejemplo, si quiero trasmitir un "saber" a otra persona, primero lo hago consciente, lo incorporo como conocimiento. Finalmente, se hace referencia a un conjunto de saberes, clases de saberes (FEYERABEND, 1974). Con esta idea se identifica un conjunto ordenado y sistematizado de conocimientos que cuentan con una lógica racional interna y con una estructura jerárquica interna. Es un conjunto de saberes, por ejemplo, el saber científico social, el saber científico natural, el saber popular ganadero, el saber popular de la carpintería, el saber mágico de los chamanes, etc. Este concepto de conjunto de saberes intenta dar cuenta de elementos fundamentales en la construcción de conocimiento: una determinada lógica-racional, la transmisibilidad de los saberes, y las características socio-históricas de la elaboración del discurso (FOUCAULT, 2000).

Esta distinción se vuelve pertinente por lo siguiente: el conjunto de saberes populares incorpora a su estructura de saberes al conocimiento científico, pero lo hace bajo sus propias reglas y valores. Por lo tanto, diferenciar conocimiento científico de saber popular no es suficiente para comprender las lógicas racionales del aprendizaje y práctica de las personas. Hay saberes populares y saberes científicos, y por otra parte hay conjuntos de saberes populares y conjuntos de saberes científicos. Cada uno de estos conjuntos de saberes tiene sus valores, sus mecanismos de generación y de validación, de registro y de trasmisión. Este primer concepto conlleva la noción de que no existe un único conocimiento ni una única forma de validarlo.

Conviene aquí dar cuenta de qué se entiende por el conjunto de saberes populares.

\begin{abstract}
Por ciencia popular -o folclor, saber o sabiduría popular- se entiende el conocimiento empírico, práctico, de sentido común, que ha sido posesión cultural e ideológica ancestral de las gentes de las bases sociales, aquel que les ha permitido crear, trabajar, e interpretar predominantemente con los recursos directos que la naturaleza ofrece al hombre (FALS BORDA, 1980).
\end{abstract}

Por otra parte, conviene señalar que, a los efectos de este trabajo, el conocimiento entendido como abstracción tiene la finalidad de transformar una realidad determinada. "El conocimiento es acción y su verdad se mide por las consecuencias útiles que implica, y la transformación crítica de la realidad en la 
resolución de problemas del individuo o de un colectivo social. Si no es así ¿para qué nos sirve el conocimiento?" (CHACíN et al., 2007).

Esta realidad que se quiere transformar es interpretada bajo los mismos lineamientos éticos, normativos y cognitivos con que interpretamos la forma de generar conocimiento, esto implica que cada persona reconoce una cierta ontología y a partir de dicha ontología elabora una epistemología. ${ }^{3}$

\begin{abstract}
Toda teoría -explica Feyerabend-, en virtud de su ontología, no solo define un modo particular de ver la realidad, sino que establece una manera de seleccionar, disponer y explicar la evidencia o hechos observacionales, fija la significación que adquieren los términos teóricos y observacionales, crea los instrumentos de observación y medición, y codifica los modos en que los resultados deben interpretarse. [...] Una teoría es incompatible con otra si sus consecuencias ontológicas son incompatibles con las consecuencias ontológicas de la última (FEREYABEND, 1962 en GARGIULO, 2016).
\end{abstract}

La ciencia positiva, herencia de la física newtoniana y de la sociología de Comte, busca transformar una realidad que es interpretada como un sistema lineal y ajeno al sujeto (ADORNO, 1972 [2001]; REALE y ANTISERI, 1988; MOULINES, 1979). De esa forma, el conocimiento no se construye, se obtiene, y analíticamente se interpreta buscando las leyes que regulan ese sistema lineal. Tal descubrimiento (las leyes) permite predecir el comportamiento de las partes del sistema (siguiendo una lógica cartesiana). Si comprendemos el comportamiento de la naturaleza y/o de la sociedad, podemos transformarla, dominarla (al estilo Bacon). Esta ciencia positiva comparte elementos ontológicos con algunos conjuntos de saberes tradicionales o metafísicos. El derecho canónico, por ejemplo, no difiere en sus intenciones de dominar la naturaleza y las sociedades a partir de una comprensión ilustrada de las leyes divinas. Al igual que en la ciencia positiva, solo algunas personas pueden comprender el significado de las leyes universales que rigen el sistema. ${ }^{4}$

En similar forma, quienes creen que la realidad no es lineal, sino que es compleja, también entienden que la forma de conocer esa realidad es a través de sistemas complejos, con múltiples variables y con determinadas incertidumbres. Entienden que el resultado de un sistema complejo no es predecible pues el orden de los factores sí altera el producto. Por ejemplo, la Teoría del Caos (Vázquez et al., 2001) y la magia de los chamanes ${ }^{5}$ : ambos casos parten de un determinado conocimiento regular, estable y sistemático, para afirmar que la realidad puede ser transformada en infinitas opciones, y por tanto que es poco probable predecirla.

Si bien puede ser una redundancia conviene aclarar que la ciencia positiva no dialoga con el derecho canónico, y que la Teoría del Caos difícilmente pueda intercambiar algún concepto con la magia de los Chamanes. Estas diferencias no son

\footnotetext{
${ }^{3}$ Este análisis puede encontrarse de forma clara en Nietzsche (1882, 1883), Bachelard (2004), Fleck (1986), Popper (1985), Bunge (1975), Feyerabend (1986, 2001), Foucault $(1968,1973,2000)$.

${ }^{4}$ Artículo 762 del Código de Derecho Canónico: "Como el pueblo de Dios se congrega ante todo por la palabra de Dios vivo, que hay absoluto derecho a exigir de labios de los sacerdotes, los ministros sagrados han de tener en mucho la función de predicar, entre cuyos principales deberes está el de anunciar a todos el Evangelio de Dios." Comparable situación de similitud epistemológica puede encontrarse entre algunos mitos de la Iglesia Católica y el empirismo, véase Feyerabend, 1986, p. 28-29.

5 "Es así como aparece toda una imaginería mental con preguntas y respuestas y a la vez con consciencia dialógica para modificar el desorden. Aquí también opera la regresión pasando por los laberintos inconscientes en que se descubre así mismo como ser, esencia y totalidad, y con ello la introversión, centrando la consciencia en el 'sí mismo' o mismidad ('self') lo que equivale a la profundidad de la esencia y existencia del ser en su microcosmos revertiéndose en su perspectiva en forma alterna y global (tiempo-espacio o témporo-espacial); así se llega a un orden, desorden y caos con la sensación del todo y/o para todos". (SÁNCHEZ, s/d).
} 
metodológicas, sino que son epistemológicas. Sin embargo, la Teoría del Caos, al parecer regida por los mismos valores sociales y culturales que la ciencia positiva, tampoco puede dialogar con esta, pues mantiene diferencias ontológicas. Lo mismo sucede con la magia de los chamanes y con el derecho canónico.

Es de esta forma que la realidad que queremos transformar está previamente delimitada y construida por un conjunto de arreglos éticos, morales, culturales y cognitivos. Los mismos arreglos que guían al conocimiento son los que nos muestran determinada realidad. Ergo, diferentes ontologías impiden el diálogo.

Finalmente, podemos también analizar propuestas teóricas o conjuntos de saberes que comparten una misma base ontológica y epistemológica, pero que difieren en sus perspectivas metodológicas. Por ejemplo, el Comunismo Científico de Marx y Engels (Engels, 1880) con la Teoría Crítica de la Escuela de Frankfurt (Adorno, [1972] 2001), y esta con la Educación popular de Paulo Freire (1973); y esta con la Investigación Acción Participación de Fals Borda (1986); y esta con la ecología de saberes de De Sousa Santos (2012). En fin, ante unas mismas bases ontológicas y con una misma o similar perspectiva epistemológica las propuestas metodológicas han sido variadas y variantes.

El primer argumento de este trabajo entonces refiere a que la posibilidad de que dialoguen dos conjuntos distintos de saberes está atravesada al menos por tres dimensiones: una ontológica, una epistemológica y una metodológica. A su vez estas dimensiones están atravesadas por componentes éticos, políticos e ideológicos; una determinada forma de ver y entender el mundo conlleva una determinada forma de conocerlo, y, por ende, de dominarlo ${ }^{6}$.

"Saber y poder, no son más que una rejilla de análisis. Vemos también que esta rejilla no está compuesta de dos categorías de elementos extraños entre sí, que serían el saber por un lado y el poder por el otro -lo que les haría exteriores entre sí. (FOUCAULT, 1995, en KARCZMARCZYK y RODRÍGUEZ, 2011).

\section{CONOCIMIENTO Y TECNOLOGÍA EN LA GANADERÍA URUGUAYA}

Mucho se ha hablado, dentro del paradigma crítico del conocimiento, sobre los diálogos de saberes, al punto de que en algunos casos se ha generado una categoría epistemológica que refiere a ese sentido: "la ecología de saberes".

En concreto, aquí se analizará el posible diálogo, en términos epistemológicos, del conjunto de saberes técnico-científicos de las ciencias naturales aplicado a la producción ganadera con el conjunto de saberes populares de la ganadería. Más específicamente, el conocimiento científico de las ciencias naturales aplicado en las disciplinas Agronomía y Medicina veterinaria con el saber popular de los/as ganaderos/as familiares.

El supuesto de base, que se quiere analizar críticamente, es el siguiente: las personas dedicadas a la ganadería familiar acumulan y trasmiten un determinado conjunto de saberes populares sobre el comportamiento de la naturaleza; generalmente este saber popular es sistematizado y trasmitido de forma oral entre las diferentes generaciones. Como se ha señalado anteriormente el saber popular es el

\footnotetext{
${ }^{6}$ Intentando respetar la extensión del artículo se vuelve difícil profundizar sobre las formas de dominación social y la producción de conocimiento. Para el caso de la ganadería familiar, lo cual se aplica también a otras formas de producción agropecuaria con racionalidades distintas, existe una serie de variables materiales, económicas, culturales y simbólicas que habilitan la producción de conocimientos, así como las resistencias a ellos. A partir de la implementación del capitalismo financiero y particularmente del desarrollo global del modelo de agronegocios, las instituciones encargadas de generar conocimientos técnicos se han dedicado a producir y desarrollar tecnologías que intentan transformar los sistemas productivos considerados "atrasados". Estas relaciones económicas y de poder existentes entre quienes generan tecnologías y las familias productoras interviene y forma parte constitutiva de los procesos de diálogo, de incorporación o de resistencia.
} 
conjunto de saberes y conocimiento más extendido, el minoritario es el saber técnico, el conocimiento científico. Esta mayoría desalineada de las reglas del mundo de la ciencia pone en marcha su vida más allá del conocimiento científico, vive en base a su saber práctico popular. Junto con ello, este saber popular intercambia, en mayor o menor medida, con un saber técnico y científico que proviene de la interacción con profesionales de la Agronomía y de la Medicina veterinaria. Bajo determinada teoría, el saber popular es un conocimiento metafísico, es doxa, o sea, improbable y basado en supuestos no verificables ni negables, mientras que el conocimiento técnico se basa en la ciencia hegemónica moderna y en el experimento impoluto. ¿Pues entonces, cómo pueden dialogar?

El conjunto de saberes técnico-científicos suele incorporarse en la época de la modernidad bajo la modalidad de sistemas expertos (GIDDENS, 1993). Estos sistemas son dispositivos (materiales, cognitivos, inmateriales) que las personas incorporan como válidos sin conocer ni comprender, pero a los cuales les depositan cierta confianza por la autenticidad del conocimiento experto que aplican. Por ejemplo: la temperatura y el pronóstico del tiempo forman parte del sistema experto de la meteorología. Muchas personas pueden mirar el pronóstico del tiempo y ver que la temperatura va a bajar en la tarde, y por tanto llevar un abrigo cuando salen a la mañana. Necesariamente no deben conocer algo sobre termodinámica, pero confían en la fuente de ese saber. En definitiva, el conjunto de saberes técnico-científicos forma parte de la vida cotidiana de las personas y en muchas ocasiones se aparece en forma de dispositivos o tecnologías. Por tecnología se refiere a la ciencia aplicada, pero como ciencia aplicada bajo determinantes socio-históricas. La tecnología, producto de la institución ciencia, no es impoluta, ni objetiva, ni neutral. "Si la tecnología es un producto y fenómeno social, la demarcación del concepto no puede desligarse del análisis de los procesos sociales, ni comprenderse aislando algunos factores y sin considerar una teoría sobre la sociedad y la raíz de sus contradicciones" (HERRERA, 1990). Bajo esta óptica la tecnología es producto de un conjunto determinado de saberes, sea el científico, sea el popular, sea el mágico, o cual sea. ${ }^{7}$ "En efecto, la ciencia y la tecnología son manifestaciones — si bien no las únicas- de la creatividad e intelecto del ser humano en su discurrir histórico" (LARA, 2009).

En este marco, se ha visto que en otros trabajos se ha abordado el estudio del comportamiento de las personas dedicadas a la ganadería del Uruguay en cuanto a la incorporación de tecnología (Morales, 2011; Gutiérrez et al., 2011; Gómez, 2011; Gómez y Saravia, 2013), en cuanto a las formas de aprendizaje (Dogliotti et al., 2012; Aguerre y Albicette, 2018), en cuanto al vínculo con la asistencia técnica y en cómo es percibida (Chía et al., 2003, De Hegedüs y Gravina, 2011; De Hegedüs, 2011). Algunas de las principales conclusiones de estos trabajos refieren a la racionalidad distinta de la producción familiar respecto a la empresarial (Morales, 2011; Gutiérrez et al., 2011; Gutiérrez y Modernel, 2011; Chía et al., 2003; García et al., 2011), sostienen que los procesos de aprendizaje son de base inductiva y con un peso importante de la experimentación empírica (Morales, 2011; Dogliotti et al., 2012; Aguerre y Albicette, 2018), concluyen además que existe un reconocimiento y un interés ante la asistencia técnica (De Hegedüs y Gravina, 2011; De Hegedüs, 2011; Gómez, 2011); en algunos trabajos se vincula la capacidad de innovación a variables estructurales de difícil modificación como la extensión en hectáreas de la explotación, la edad de quien dirige la empresa, la infraestructura predial, y finalmente la motivación (ECP, 1991; Gómez, 2011); en otro trabajo del Instituto Plan Agropecuario, De Souza

\footnotetext{
${ }^{7}$ Las pirámides asignadas al pueblo Maya son un buen reflejo de la aplicación de tecnologías de construcción basadas en un saber popular (al que posteriormente intenta fundamentar el saber científico) y que al mismo tiempo tienen componentes de un saber mitológico o mágico.
} 
(2009) afirma que, según un estudio realizado, nueve de cada diez familias entienden que los cambios dentro de las explotaciones/empresas se deben a factores familiares y/o sociales (DE SOUZA, 2009). No obstante, los procesos de extensión y de transferencia se perciben aún como insuficientes, "muchas tecnologías de proceso, de relativamente fácil implementación y reducido costo, muestran aún baja adopción" (GÓMEZ, 2017).

Ante este tipo de diagnósticos la academia se ha abocado a la generación de métodos y metodologías de extensión que permitan superar esas barreras culturales y que a fin de cuentas las familias ganaderas incorporen nuevas tecnologías o generen prácticas innovadoras. Si bien en los últimos años estas nuevas propuestas han cambiado en sus formas metodológicas, y algunas epistemológicas, aún mantienen la propuesta original de la difusión de innovaciones: llevar una propuesta concreta de innovación tecnológica a ser incorporada por las familias. En estos casos las propuestas metodológicas y pedagógicas son variantes para que las familias incorporen tecnologías (de insumos o de procesos) que son previamente establecidas. Lo que cambia es la metodología, pero el objetivo de difusión y transferencia se mantiene.

La posición difusionista da un lugar central a la innovación y apunta a la adopción por parte del/la productor/a. En el difusionismo el conocimiento es producido en centros de investigación, privados o públicos, y tiene una fuerte orientación aplicada. En consecuencia, prima en programas de extensión de transferencia tecnológica donde el extensionista es una fracción de un proceso de ciencia-innovación-aplicación. Se asienta sobre una concepción del cambio focalizado en la posición individual de cada productor/a (FLORIT, PIEDRACUEVA, 2018).

En el presente trabajo se asume que el conocimiento es situado, que es construido y que se enmarca en un conjunto más amplio de saberes. En esta estructura no hay lugar para la difusión de innovaciones. Una tecnología $X$ sobre el manejo del rodeo no puede ser difundida y adoptada; esa tecnología ingresará en una estructura más amplia, ingresará en un sistema complejo habitado por personas, recursos, saberes, creencias, intereses, expectativas, fracasos, memorias; esa tecnología se pondrá en diálogo con otros saberes y surgirá una nueva tecnología, una adaptada a ese sistema.

Dadas esas condiciones es que se vuelve necesario incorporar al análisis de las prácticas de extensión una dimensión epistémica sobre los conjuntos de saberes populares de la ganadería 8 .

La dimensión epistémica y cognitiva que se propone para el análisis refiere a las formas de validación del conocimiento que rigen en una estructura de saber popular. Es fundamental, a estos efectos, conocer de qué forma las personas vinculadas a la ganadería construyen y validan el conocimiento práctico, así como también el conocimiento científico.

\footnotetext{
${ }^{8}$ Recordemos que los conjuntos de saberes pueden ser analizados a través de tres dimensiones (una ontológica, una epistemológica y una metodológica) y que estas dimensiones se encuentran atravesadas por variables estructurales (económicas, lingüísticas, políticas) así como por variables simbólicas (historias locales, tradiciones orales, creencias).
} 


\section{LA ECOLOGÍA DE SABERES COMO SOPORTE DE LA TRADUCCIÓN}

La ecología de saberes se ofrece como una herramienta teórica y política frente a una de las características de la racionalidad eurocéntrica: la monocultura del saber y del rigor del saber. Según De Sousa Santos la sociología de las ausencias nos permite identificar cinco características de la racionalidad eurocéntrica que invisibilizan a todo aquello que se opone a sus valores ético-políticos: monocultura, universalismos, productividad, globalidad, discriminación y colonialismo. Bajo esta concepción la extensión es entendida como comunicación o como extensión de contrario (MONTEIRO NEVES y BENINCÁ, 2018).

Ante este panorama De Sousa Santos propone una alternativa que permita resignificar las no existencias: la ecología de saberes. "Neste dominio, a sociologia das ausências tem por objetivo substituir a monocultura do saber científico por uma ecologia de saberes" (DE SOUSA SANTOS, 2002). La idea central de esta categoría recae en su concepción de convivencia y complementariedad. La ecología de saberes apela a un concepto de la biología clásica para dar cuenta de que no existe una única especie de saber o conocimiento, sino que estas formas conviven y se complementan con otras formas de saber y conocer el mundo. Ahora bien, la construcción de un sistema de saberes se enfrenta con una serie de problemas de significados que implican varios aspectos. Por una parte, podemos enfrentarnos a un fenómeno de inconmensurabilidad (tanto en el sentido dado por Kuhn como en el sentido propuesto por Feyerabend); nos enfrentamos a una cuestión de lenguas y dialectos; nos enfrentamos a cuestiones de construcciones simbólicas y pujas culturales; nos enfrentamos a una lucha de intereses ${ }^{9}$.

De Sousa Santos propone a la "traducción intercultural" como un mecanismo de apoyo ante estas características. La traducción es un ejercicio recíproco, esto es que solamente tiene sentido cuando todos los saberes tienen cierta disposición a realizar un ejercicio de traducción. Esta disposición implica, en cierto modo, una confluencia previa de intereses. Sin dudas que esta confluencia de intereses difícilmente esté marcada por el saber en sí mismo, generalmente la confluencia se da por problemáticas comunes que poco tienen que ver con el saber, con el aprendizaje o con la epistemología. La traducción en términos de ecología se da en modo de diálogo horizontal entre saberes, tarea nada sencilla, porque el saber es en sí mismo una relación de poder.

"Como una ecología de saberes, el pensamiento posabismal se presupone sobre la idea de una diversidad epistemológica del mundo [...] esto implica renunciar a cualquier epistemología general" (DE SOUSA SANTOS, 2010). Las epistemologías generales van de la mano de las teorías generales, parten de la base de una monocultura interpretativa. Así como existe una única forma de conocer la verdad, hay una única forma de comprenderla. Esta ontología no hace más que ocultar una parte importante del mundo, no hace más que producir constantemente ausencias. Sin dudas que será imposible establecer un diálogo de saberes entre ciencia y saber popular cuando se parte de esta ontología, puesto que quienes adhieren a las

\footnotetext{
${ }^{9}$ En los procesos de construcción de conocimiento, y por tanto en los posibles diálogos de saberes, las relaciones de poder son parte constitutiva. Estas relaciones pueden darse en términos de dominación simbólica, en términos de dominación de clases, en términos de dominación cultural o territorial. Se asume que dos sujetos sociales distintos que ocupan diferentes posiciones en la estructura social cuentan con intereses disímiles e incluso antagónicos. Esta relación habilita mecanismos de dominación pero también de resistencia; así sucedió con los saberes ancestrales de la naturaleza en América y su apropiación por parte de las academias europeas; así sucede también con la generación de patentes de la industria farmacéutica sobre las propiedades medicinales de algunas plantas utilizadas por las poblaciones locales del continente americano; y también con los paquetes del agronegocio que pretenden imponerse sobre las prácticas productivas del campesinado.
} 
verdades únicas ni siquiera contemplan la posibilidad de dialogar con otros saberes, porque estos no existen.

Para la ciencia hegemónica el saber popular no es un tipo distinto de conocer y acumular conocimiento, es un saber vulgar, no cierto, y que aún no ha llegado a desarrollarse hasta comprender lo que le otorga el conocimiento científico. El saber popular es, para la ciencia, lo que el subdesarrollo es para las teorías generales del desarrollo económico. Como señala De Sousa Santos (2010) la ciencia hegemónica, como tal, solamente analiza sus límites y restricciones dentro la propia ciencia. Así se ha desarrollado la epistemología tradicional, la filosofía de la ciencia e incluso algunas vertientes críticas de la epistemología que analizan a la ciencia como una institución social, pero que se limitan a estudiar solamente el campo de la ciencia. En este contexto, el uso contrahegemónico de la ciencia solamente tiene sentido en un marco más amplio, por ejemplo, en el de una ecología de saberes (DE SOUSA SANTOS, 2002).

\subsection{UNA ECOLOGÍA DE LOS SABERES EN LA GANADERÍA: LAS AUSENCIAS}

La ecología de saberes requiere del reconocimiento explícito de al menos dos formas distintas de conocer e interpretar el mundo, si no existe ese mutuo reconocimiento pues entonces no existe la posibilidad del diálogo. Una de las características de la ciencia, y por ende de las prácticas de extensión, es el no reconocimiento de otras formas de conocimiento como interlocutores válidos.

Las prácticas tradicionales de extensión interpretan que existe un conocimiento vulgar o popular que debe ser superado, que debe ser "evolucionado", que debe ser "convencido". Las prácticas tradicionales de extensión no cuestionan la forma en que se genera el conocimiento científico, no cuestionan si es útil, si es pertinente; cuestionan las formas en que ese conocimiento puede ser adoptado; lo que está en juego, en todo caso, es la práctica, pero no el conocimiento.

Las prácticas de extensión son analizadas bajo las lógicas internas de la ciencia; las nuevas metodologías de extensión deben hacerse conforme a las reglas de la institución ciencia moderna: deben ser objetivas, escalables, productivas, predictivas. Con estos criterios quedan por fuera todas aquellas prácticas de extensión que incorporen otras formas de conocer, que no puedan reproducirse de forma masiva, que no redunden resultados económicos directos, que no logren predecir qué es lo que va a suceder con el sistema.

De forma similar, las prácticas de extensión generan ausencias en relación a las prácticas de las familias ganaderas. Aquellas prácticas que realizan las familias y que no cuentan con un soporte científico son, en principio, desestimadas o subvaloradas (por ejemplo, identificar la preñez de una vaca por el pelaje, o la enfermedad de una oveja por el lugar donde come); también se generan ausencias con aquellas prácticas que no generan un resultado económico directo y que son catalogadas como improductivas (como apostar a la producción ovina de carne, o tener más caballos de los que "se necesitan" en el campo); se generan ausencias cuando se identifica una práctica específica que le dio "resultado" a una familia, pero que no puede ser trasladada a otras familias, si solo le sirvió a esa familia entonces tampoco es útil (por ejemplo, las estimaciones climáticas que realizan en base al comportamiento de sus animales o por la observación del rocío en su predio, o las nubes desde su casa); se generan ausencias cuando se identifican acciones que dieron "resultado" un año pero que para el próximo no sirvieron, porque lo válido es lo lineal, lo que permite predecir el funcionamiento del sistema.

Muchas de estas prácticas se vuelven invisibles pues no se amoldan con las metodologías de extensión y difusión de innovaciones. La extensión tradicional 
propone, por ejemplo, que las familias logren mejorar el porcentaje de preñez de las vacas y para ello necesitan que las familias incorporen en sus hábitos las prácticas de la ciencia: control riguroso de los procesos, registro, análisis, gestión, medición, evaluación. No hay posibilidad de diálogo entre los saberes si la familia lleva el control del porcentaje de preñez en base a cómo se ve el ganado y a cómo se comportan las vacas; la ciencia necesita datos científicos para poder dialogar.

El conjunto de saberes populares de la ganadería es estrictamente subjetivo en tanto se genera a partir de un sujeto específico. El conocimiento de que las "nubes azules" anuncian frío no fue adquirido de una revista, de un programa de radio, no fue adquirido de una charla técnica; fue construido con el paso de los años mediante la observación directa de la persona al cielo tal y como se ve desde su lugar, desde su hogar. El conocimiento de que se avecina una tormenta porque el ganado "está nervioso" se basa en un saber situado y concreto, no es cualquier ganado, no es cualquier tipo de estado nervioso.

La ecología de saberes entiende al conocimiento como intervención de la realidad y no como representación de la misma (DE SOUSA SANTOS, 2010). Al conocer una parcela de la realidad la estamos al mismo tiempo transformando y, por lo tanto, el sujeto que conoce, cómo conoce, con quiénes lo hace, dónde, cuándo, etc., son factores determinantes. Con esto no debe entenderse que no se reconozcan condiciones materiales y estructurales de existencia que puedan performar las formas de conocer, no es eso. Es que aun esas condiciones materiales de existencia, al ser conocidas, son incorporadas, interpretadas y transformadas por un sujeto social histórico particular.

Ante ese esquema de construcción de conocimiento, construido mediante la experiencia subjetiva y que implica muchos años, es complejo el diálogo con saberes técnicos que no implican un sujeto cognoscente, que son inmediatos, transportables, ajenos.

La cuestión aquí no es invalidar el tacto o la ecografía como fuente de información para determinar la preñez de una vaca, o el uso de información satelital para conocer las probabilidades de que baje la temperatura; la cuestión es analizar de qué forma se puede favorecer un diálogo efectivo de saberes que no implique la anulación forzosa del saber popular.

De Souza Santos propone el concepto de pensamiento abismal para comprender estas dinámicas. Según el autor el pensamiento occidental es abismal en el sentido de que genera una línea simbólica de separación entre lo válido y lo irrelevante, entre lo existente y lo inexistente.

\footnotetext{
Una división en la que "el otro lado de la línea" se desvanece como realidad, se convierte en no existente, y de hecho se produce como inexistente. No existente significa que no existe de ninguna forma relevante o comprensible de ser. Todo lo que se produce como no existente se excluye radicalmente, porque se encuentra más allá del reino de lo que la concepción aceptada de inclusión considera que es su otro (DE SOUSA SANTOS, 2017).
}

Esta división puede manifestarse cuando el/la técnico/a intenta anular el conocimiento de la familia ganadera, cuando intenta desprestigiar ese conocimiento y la forma en que este fue construido.

Pero también se manifiesta cuando los/as profesionales que visitan a una familia ni siquiera se dan cuenta de que ese conjunto de saberes populares existe; se manifiesta cuando visitan un predio, pero con una mirada obtusa. No es que intentan anularlo, es que ni siquiera saben que existe. 
La hipótesis que aquí se sostiene es que el "des-conocimiento"10 de los componentes cognitivos y epistémicos del saber popular de la ganadería familiar resulta en una barrera efectiva ante un intento de diálogo de saberes, y por tanto, aun contando con nuevas propuestas metodológicas y pedagógicas, el diálogo de saberes se ve interrumpido, y con ello se interrumpe la puesta en diálogo de determinada tecnología.

\title{
4. LOS CONJUNTOS DE SABERES COMO ESQUEMAS DE INTERPRETACIÓN
}

Se planteó con anterioridad que un conjunto de saberes es entendido como un conjunto ordenado y sistematizado de conocimientos que cuentan con una lógica racional interna y con una estructura jerárquica interna. Es un conjunto de saberes en tanto dentro de ese esquema coexisten y dialogan diversas formas de saber y diferentes conocimientos. Lo que caracteriza y distingue a un conjunto de saberes de otro, no es el saber en sí mismo, no es el conocimiento; lo que los distingue es la forma en que se ordenan esos conocimientos, bajo qué criterios son incorporados, con qué reglas se manejan, cuáles son las jerarquías que funcionan dentro de ese conjunto.

\begin{abstract}
Aquello que debe oponerse, en el análisis del desarrollo histórico del conocimiento científico, no es la "razón" a los "sentidos", sino determinadas formaciones racionales y sensoriales (determinadas organizaciones de las percepciones según ciertas líneas de conexión "racional") a otras formaciones que entran en conflicto con las primeras (BUENO, 1976).
\end{abstract}

Establecer qué es lo que se sabe, cuál es el conocimiento sobre un fenómeno o proceso, no depende del "tipo" de conocimiento o saber que esté en juego, sino que depende del conjunto de saberes con los cuales nos estemos manejando.

No se puede afirmar que existe el "conocimiento científico" ni tampoco que existe el "saber popular"; lo que existe es un conjunto de saberes generados de diferentes formas y validados según una determinada estructura lógica, racional y jerárquica.

Encuadrar el trabajo de extensión bajo un esquema de conjuntos de saberes también implica romper con la ilusión del puritanismo. Es falso que las familias ganaderas utilizan solamente saberes populares; en general las familias ganaderas han ido incorporando a lo largo de su historia una diversidad muy amplia de conocimientos y tecnologías producidas por la ciencia.

Al momento de pensar en términos de ecología y diálogo de saberes es fundamental reconocer que antes de la llegada del/la profesional las familias ya manejan un conjunto de conocimiento técnico-científicos, y que los han incorporado en su esquema de conjuntos de saberes populares.

Este concepto es también válido para los/as profesionales. Es falso que los/as profesionales llegan al predio portando únicamente conocimientos técnicocientíficos; es falso también que los conocimientos que ponen en juego sean únicamente técnico-científicos.

En primer lugar, porque los/as profesionales son, antes que todo, personas y seres humanos comunes y corrientes, que piensan, que sienten, que tienen intereses, que tienen apuros, que tienen expectativas; en segundo lugar, porque los conocimientos técnico-científicos que manejan también interactúan (dentro de sí) con

${ }^{10}$ Este desconocimiento forma parte constitutiva de la producción de ausencias señalada por De Sousa Santos. 
otros saberes y conocimientos que fueron generados por la experiencia; $y$, en tercer lugar, porque los conocimientos técnico-científicos son falibles y cambiantes.

Así como puede juzgarse que un productor ganadero hace un uso arbitrario de un conocimiento técnico (por ejemplo, cuando "automedica" a sus animales); también es cierto que un ingeniero agrónomo realiza un uso arbitrario de sus conocimientos cuando recomienda a un productor tal o cual medida, tal o cual insumo, sin realizar el trabajo de diagnóstico necesario: por ejemplo recomendar el uso de fertilizantes sin un análisis de suelo (algo similar a lo que Bourdieu et al., 2002, refieren como la "sociología espontánea"). Ambos apelan a su experiencia sensorial y práctica para poner en juego un determinado conocimiento, sea este científico o popular.

En definitiva, más que el "tipo" de conocimiento lo que interesa es el conjunto de saberes dentro del cual ese conocimiento entra en juego. Interesa comprender cuál es la racionalidad de ese conjunto de saberes, cuáles son sus reglas, cuáles son los criterios de validación, cuáles son los usos permitidos. Reconocer estos sistemas forma parte de una aproximación a una ecología de saberes, a una forma de poner en diálogo diferentes formas de conocer e interpretar el mundo.

A este esquema complejo de diálogo entre conjuntos de saberes distintos debe agregarse una de las características intrínsecas de la gnoseología y de la ciencia como disciplina: la falibilidad y la heterogeneidad. Si bien en los manuales la ciencia se presenta como una aproximación universal y homogénea a la verdad, en la práctica los conocimientos científicos distan mucho de ese anhelo.

La epistemología tradicional y la epistemología crítica han logrado argumentar que los mecanismos objetivos de la ciencia moderna son prácticamente inexistentes; se ha argumentado también que la ciencia no puede aspirar a la verdad (entendida como correspondencia) pues los mecanismos (metodológicos e institucionales) no podrán proveer certeza alguna sobre ese resultado. Se ha argumentado que la ciencia es, por definición, falible: son falibles sus mecanismos, sus métodos y por tanto sus resultados.

\begin{abstract}
En un análisis más minucioso se descubre que la ciencia no conoce "hechos desnudos" en absoluto, sino que los "hechos" que registra nuestro conocimiento están ya interpretados de alguna forma y son, por tanto, esencialmente teóricos. Siendo esto así, la historia de la ciencia será tan compleja, caótica y llena de errores como las ideas que contiene, y a su vez, estas ideas serán tan complejas, caóticas, llenas de errores y divertidas como las mentes de quienes las han inventado (FEYERABEND, 1986).
\end{abstract}

Las prácticas tradicionales de extensión cargan consigo un conocimiento científico, lo hacen en un conjunto de saberes científicos, pero ese conocimiento es falible. Sin embargo, cuando un equipo de extensionistas sale al campo portando esos conocimientos lo hacen convencidos de que llevan un conocimiento certero, fiable. Los/as científicos/as y extensionistas parten de la premisa de que el conocimiento científico es fiable y se generan mecanismos de difusión a partir de esa fiabilidad. "En todo juicio de validez científica permanece así implícito el supuesto de que aceptamos las premisas de la ciencia y que la conciencia del científico es algo en que puede confiarse" (POLANYI, 1946).

Sin embargo, esta fiabilidad no resiste análisis alguno. Si se analizan las tecnologías y conocimientos difundidos desde la ciencia hacia la ganadería en los últimos cincuenta años, se puede observar con facilidad la falibilidad de la ciencia; se puede observar con facilidad la no fiabilidad, se puede observar cómo la ciencia y la extensión han difundido tecnologías que no solo no logran alcanzar los resultados 
previstos, sino que han generado otros resultados no esperados y negativos. Como ejemplo puede manejarse la difusión de las pasturas sembradas como una medida de "desarrollo" hace unos veinte años y cómo hoy se difunden tecnologías de manejo del campo natural y se recomiendan reducir las áreas de pasturas "mejoradas"; la promoción de la siembra directa y del uso de herbicidas y cómo hoy se promueven prácticas de transición agroecológicas.

Esta característica de la ciencia implica también que no existan propuestas universales y homogéneas. En un proceso de difusión se intentará anular un conocimiento popular sobre el manejo de las "malezas" y se recomendará la utilización de herbicidas; en otro se recomendarán medidas de manejo del campo natural de forma extensiva; en otro se recomendará el sistema racional de pastoreo Voissen; en otro se recomendará la incorporación de burros; en otro se recomendará que se baje la dotación de ovinos; etc. ¿Con qué argumento la ciencia desplaza los saberes populares si dentro de la misma ciencia existen diversos conocimientos y saberes?

¿Cómo dialoga la ciencia a su interna con todos los conocimientos científicos? ¿Cómo dialogan las disciplinas científicas? ¿Cómo, en ese contexto, se puede generar una ecología de saberes?

La tabla $\mathrm{N}^{\circ} 1$ intenta, de forma muy esquemática, agrupar algunos de los criterios de validación del conjunto de saberes científicos (tomando en cuenta las producciones de la sociología de la ciencia, la epistemología y sus corrientes críticas). De la misma forma, con base en la revisión teórica de las propuestas de Investigación Acción Participación, Educación popular y ecología de saberes, y a partir de la revisión de los documentos empíricos seleccionados sobre el caso uruguayo, se intentan identificar aquellos criterios de validación que pueden estar formando parte del conjunto de saberes populares de la ganadería.

Tabla 1 - Aproximación a criterios de validación

\begin{tabular}{|l|l|}
\hline Conjunto de saberes científicos & Conjunto de saberes populares \\
\hline Metódico & Sistemático \\
\hline Numérico & Oral \\
\hline Riguroso & Tradicional \\
\hline Transportable & Situado \\
\hline Ajeno & Sensorial \\
\hline Producción rápida & Construcción lenta \\
\hline Sectorial & Complejo \\
\hline
\end{tabular}

Fuente: Elaboración propia con base en la revisión teórica y de los documentos empíricos seleccionados.

\section{LA TRADUCCIÓN INTERCULTURAL EN LA GANADERÍA FAMILIAR}

La traducción intercultural que se propone en este trabajo se enmarca en el diálogo de dos conjuntos de saberes: el popular ganadero y el científico ganadero. Las racionalidades, valores, reglas, criterios, epistemologías, lenguajes de estos dos conjuntos generan una estructura de inconmensurabilidad. Ante esta situación, como se ha visto con anterioridad, culturalmente se impone el conjunto de saberes científicos.

La ecología de saberes propone que

Siempre que existan intervenciones del mundo real que puedan, en teoría, ser implementadas por diferentes sistemas de conocimientos [...] la preferencia debe ser dada a la forma de conocimiento que garantice el mayor nivel de participación a los grupos sociales involucrados en su diseño, ejecución y control, 
y en los beneficios de la intervención (DE SOUSA SANTOS, 2010).

Para que pueda darse preferencia a un conjunto de saberes determinado en primer lugar deben generarse las condiciones para que ese conjunto sea reconocido. Es en ese marco que la sociología de las ausencias y de las emergencias cumple un rol fundamental. Las prácticas de la extensión en la ganadería deben generar mecanismos que permitan poner en visibilidad aquellas prácticas y saberes que han sido ocultos e invisibilizados por la ciencia moderna, por el conjunto de los saberes científicos de la ganadería.

Este proceso no es natural, no es mecánico. Los equipos de extensionistas muy lejos están aún de contar con una formación académica que le sugiera (siquiera) que existen prácticas de invisibilización de otros saberes; lejos están de contar con una formación crítica sobre la sociología de las ausencias, sobre la sociología de las emergencias.

La traducción es el procedimiento que permite crear inteligibilidad recíproca entre las experiencias del mundo, tanto las disponibles como las posibles, reveladas por la sociología de las ausencias y la sociología de las emergencias (DE SOUSA SANTOS, 2002).

La traducción es el ejercicio de la puesta en diálogo, no es un ejercicio técnico, es un ejercicio político porque la traducción es política. No se traducen palabras, se realiza un ejercicio de traducción entre cosmovisiones distintas, entre intereses distintos, costumbres distintas, lenguajes distintos. El ejercicio de traducción se realiza en un marco de situaciones de dominación, en situaciones donde un modelo de producción con base en ciertos conocimientos científicos se enfrenta a otro modelo de producción con base en saberes populares.

Cuando el equipo de extensión intenta difundir conocimientos científicos hegemónicos no hace otra cosa que intentar transformar los sistemas de producción familiares en sistemas empresariales; intenta que la racionalidad de la ganadería familiar sea sustituida por la racionalidad empresarial. Los conocimientos científicos de la ganadería han sido generados con ese objetivo: aumentar la rentabilidad de la empresa ganadera.

La familia ganadera quiere vivir mejor, pero no necesariamente quiere convertirse en una empresa capitalista. El ejercicio de traducción en la extensión debe enfrentar esa lucha, debe reconfigurar los conjuntos de saberes científicos dejando de lado el supuesto de que la única forma de progreso de la ganadería familiar es que se "capitalice", que se vuelva una empresa de mercado capitalista.

Como se ha señalado a lo largo del artículo, el diálogo de saberes es posible en tanto existen condiciones materiales, políticas e ideológicas que lo permitan. Sin embargo, estas predisposiciones no son suficientes si no se cuenta con una nueva epistemología, con otras formas de validar y de construir el conocimiento. Para ello se considera fundamental identificar los criterios de validación de conocimientos en ambos conjuntos de saberes ya que, como aquí se ha intentado plasmar, el diálogo se da entre esos criterios y no en sus contenidos.

La traducción es, simultáneamente, un trabajo intelectual y político. Y es también un trabajo emocional porque presupone el inconformismo ante una carencia que depende del carácter incompleto o deficiente de un conocimiento dado o de una práctica dada (DE SOUSA SANTOS, 2002). 


\section{BIBLIOGRAFÍA}

ADORNO, T. Epistemología y ciencias sociales. Madrid: Ediciones Cátedra, 2001.

AGUERRE, V.; ALBICETTE, M. (ed). Co-innovando para el desarrollo sostenible de sistemas ganaderos familiares de Rocha - Uruguay. Serie Técnica n. 243, INIA, Montevideo, 147 p., 2018.

ÁLVAREZ PEDROSIAN, E. Crear, aprender y compartir: apuntes epistemológicos sobre la integralidad. Integralidad: tensiones y perspectivas. Cuadernos de Extensión, SCEAM-Udelar, Montevideo, n. 1, p. 61-83, 2011.

BACHELARD, G. La formación del espíritu científico. 25. ed. México: Siglo XXI Editores, 2004.

BARTABURU, D. (coord.) Evaluación de una metodología de modelación y simulación participativa para contribuir a la comprensión y comunicación del fenómeno de la sequía y mejorar la capacidad de adaptación de productores ganaderos del basalto. Plan Agropecuario, INIA, Montevideo, 44 p., 2011.

BOURIDEU, P.; CHAMBOREDON, J.; PASSERON, J. El oficio de sociólogo. 1. ed. Buenos Aires: Siglo XXI Editores Argentina, 2002.

BUENO, G. Estatuto gnoseológico de las ciencias humanas. Fundación Juan March. En Teoría del cierre categorial. Tomo I, cap. II, p. 192-236, 2011.

BUNGE, M. La ciencia. Su método y su filosofía. Buenos Aires: Editorial Siglo Veinte, 1975.

CHACÍN, B.; GONZÁLEZ, M.; TORRES, Y. Crítica a la generación del conocimiento en la extensión universitaria: aproximación a un protocolo de investigación innovativa. Laurus, Universidad Pedagógica Experimental Libertador, Caracas, v. 13, n. 24, p. 215-240, mayo/agosto 2007.

CHÍA, E.; TESTUT, M.; FIGARI, M.; ROSSI, V. Comprender, dialogar, coproducir: reflexiones sobre el asesoramiento en el sector agropecuario. Agrociencia, Facultad de Agronomía, Udelar, Montevideo, n. 1, v. 7, p. 77-91, 2003.

COMTE, A. Discurso sobre el espíritu positivo. [s/d] 1844. Versión digital disponible en: http://www.pensamientopenal.com.ar/system/files/2014/12/doctrina37229.pdf. Acceso el: 19 abr. 2018.

DE HEGEDÜS, P.; GRAVINA, V. Percepciones de productores familiares ganaderos en relación a la sustentabilidad y a la innovación. Plan Agropecuario, Montevideo, $n$. 138, p. 62-63, mayo 2011.

DE HEGEDÜS, P. Utilizando metodologías participativas de trabajo con productores: Adaptación de los ganaderos del Basalto a la sequía. En BARTABURU, D. (coord.) Evaluación de una metodología de modelación y simulación participativa para contribuir a la comprensión y comunicación del fenómeno de la sequía y mejorar 
la capacidad de adaptación de productores ganaderos del basalto. Salto, Uruguay: Plan Agropecuario, INIA, p.27-30, dic. 2011.

DE SOUSA SANTOS, B. Justicia entre saberes. Madrid: Morata, 2017. p.159-179.

DE SOUSA SANTOS, B. Introducción a las epistemologías del sur. En Construyendo las Epistemologías del Sur - para un pensamiento alternativo de alternativas. Buenos Aires: CLACSO, 2018.

DE SOUSA SANTOS, B. De las dualidades a las ecologías. La Paz: Red Boliviana de Mujeres Transformando la Economía (REMTE), serie: Cuaderno de Trabajo n. ${ }^{\circ} 18$, 2012.

DE SOUSA SANTOS, B. Para descolonizar Occidente: más allá del pensamiento abismal. Buenos Aires: CLACSO, 2010. 139 p.

DE SOUSA SANTOS, B. Descolonizar el saber, reinventar el poder. Montevideo: Ediciones Trilce, 2010.

DE SOUSA SANTOS, B. Para uma sociologia das ausencias e uma sociología das emergências. Revista crítica de Ciências Sociais. Centro de Estudos Sociais da Universidade de Coimbra, n. 63, p. 237-280, oct. 2002.

DE SOUZA, P. Factores que cambian las trayectorias de los predios ganaderos. En Morales, H.; Dieguez, F. (ed.). Familias y Campo. Rescatando estrategias de adaptación. Proyecto Integrando Conocimientos. Montevideo: Instituto Plan Agropecuario, p. 107-114, nov. 2010.

DOGLIOTTI, S. et al. Desarrollo sostenible de sistemas de producción hortícolas y hortícola-ganaderos familiares: una experiencia de co-innovación. FPTA $n .^{\circ} \mathbf{3 3}$, INIA Montevideo, 112 p., 2012.

ECP (Equipos consultores asociados). Tecnología en áreas de ganadería extensiva: encuesta sobre actitudes y comportamientos. Serie Técnica $n .{ }^{\circ}$ 14, INIA, Montevideo,1991. 98p.

ENGELS, F. Del socialismo utópico al socialismo científico. En Marx, K. y Engels, F. Obras escogidas en tres tomos. Chile: Editorial Progreso, [s/d], t. III, p. 98-160. Versión digital disponible en: http://www.archivochile.com/Ideas_Autores/engelsf/engelsde00031.pdf Acceso: 19 abr. 2018.

FALS BORDA, O. Conocimiento y poder popular: lecciones con campesinos de Nicaragua, México y Colombia. Bogotá: Siglo XXI, 1985. 175 p.

FALS BORDA, O. La ciencia y el Pueblo, nuevas reflexiones. En Salazar, M. (coord.) La investigación-acción participativa: inicios y desarrollos. España: Editorial Popular, Organización de Estados Iberoamericanos para la Educación, la Ciencia y la Cultura (OEI), Sociedad Estatal Quinto Centenario, 1992. p. 65-84.

FEYERABEND, P. Cómo defender a la sociedad de la ciencia. Polis. Revista de la Universidad Bolivariana, Santiago de Chile, v. 1, n. 1, 2001. 
FEYERABEND, P. Adiós a la razón. 3.ed. Traducción: José R. de Rivera. Madrid: Editorial Tecnos S.A., 1996. p. 1-122.

FEYERABEND, P. Tratado contra el método. Esquema de una teoría anarquista del conocimiento. Traducción: Diego Ribes. Madrid: Editorial Tecnos S.A., 1986. Capítulos 1 a 5, p. 1-52, capítulos 15 a 17, p. 157-214.

FLECK, L. La génesis y el desarrollo de un hecho científico. Introducción a la teoría del estilo de pensamiento y del colectivo de pensamiento. Madrid: Alianza Editorial, 1986.

FLORIT, P.; PIEDRACUEVA, M. Hacia una mirada transversal de la perspectiva de género en el Sistema de Asistencia Técnica y Extensión Rural. Presentación en Jornadas Nacionales de extensión, Universidad de la República, Montevideo, 12 16 nov. 2018.

FOUCAULT, M. La Arqueología del Saber. Argentina: Siglo XXI Editores, 2000.

FOUCAULT, M. La verdad y las formas jurídicas. Río de Janeiro, Pontificia Universidade Catolica do Rio de Janeiro, 1973. Versión digital disponible en: www.fmmeducacion.com.ar/Bibliotecadigital/Foucault_Laverdad.pdf.

FOUCAULT, M. Las palabras y las cosas. Una arqueología de las ciencias humanas. Buenos Aires: Siglo XXI Editores, 1968.

FREIRE, P. ¿Extensión o Comunicación? La concientización en el medio rural. 13. ed. Montevideo: Siglo XXI Editores y Tierra Nueva, 1984.

GARGIULO, T. La doctrina de la inconmensurabilidad en Paul Feyerabend: una objeción contra una particular concepción de racionalidad científica. Areté Revista de Filosofía, v. 28, n. 1, p. 61-87, 2016.

GARCÍA, R. et al. Sustentabilidad de los criadores familiares. Plan Agropecuario, Montevideo, n. 138, p. 64-68, mayo 2011.

GIDDENS, A. Consecuencias de la modernidad. Madrid: Alizana, 1993.

GÓMEZ, R. Adopción de tecnología en sistemas ganaderos del norte. Serie Técnica n. ${ }^{\circ}$ 235, INIA, Montevideo, 117 p., 2017.

GÓMEZ, R.; SARAVIA, H. Cambio técnico en sistemas ganaderos criadores de sierras del este. Serie Técnica n. ${ }^{\circ}$ 207, INIA, Montevideo, 130 p., 2013.

GÓMEZ, R. Estudio sobre la significación de la tecnología en predios familiares de ganadería extensiva de Tacuarembó (Uruguay). 2011. 171 p. Tesis de Maestría, Escuela para Graduados Ing. Agr. Alberto Soriano, Facultad de Agronomía, Universidad de Buenos Aires, 2011.

GUTIÉRREZ, R. et al. Dinámicas económico-productivas de la producción familiar criadora. Plan Agropecuario, Montevideo, n. 137, p. 62-66, mar. 2011. 
GUTIÉRREZ, R.; MODERNEL, P. Los procesos de adopción y manejo tecnológico en la producción familiar criadora. Plan Agropecuario, Montevideo, n. 140, p. 60-63, dic. 2011.

HERRERA, R. Tecnología y sociedad. Rev. Filosofía de la Universidad de Costa Rica, v. 28, n. 67/68, p. 77-84, 1990.

KARCZMARCZYK, P.; RODRÍGUEZ, N. Crítica, ideología según Michel Foucault. Cad. de Pesq. Interdisc. em Ci-s. Hum-s., Florianópolis, v. 12, n. 100, p.3-20, jan./jul. 2011.

LARA, L. Acerca de la potencialidad secuestrada de la ciencia y tecnología: Por una idea del desarrollo multidimensional. En Figueroa, S.; Sánchez, G.; Vidales, A. La ciencia y tecnología en el desarrollo: una visión desde América Latina. Zacatecas: Universidad Autónoma de Zacatecas, 2009. p. 17-22.

LÓPEZ, J. Epistemología popular: condicionantes subjetivos de la credibilidad. Revista CTS, n. 10, v. 4, p. 159-170, enero 2008.

MANRIQUE, H. Saber y conocimiento: una aproximación plural. Acta Colombiana de Psicología. Universidad Católica de Colombia. Bogotá, v. 11, n. 2, p. 88-100, dic. 2008.

MERTON, R. La sociología de la ciencia 2. Madrid: Alianza Universidad, 1977.

MONDELLI, M.; PICASSO, V. Trayectorias tecnológicas en la ganadería uruguaya: un enfoque evolucionista. 2001. 126 p. Tesis de grado, Facultad de Agronomía, Udelar, Montevideo, 2001.

MONTEIRO NEVES, F.; BENINCÁ, D. Extensão universitária popular e agroecologia no extremo sul da Bahia: campos para novas racionalidades e práticas libertadoras. Experiência, Santa Maria, v. 4, n. 2, p. 4-16, ago./dez. 2018.

MORALES, H. Aproximación global a la explotación agropecuaria. Plan Agropecuario, Montevideo, n. 137, p. 58-61, mar. 2011.

MOULINES, C. La génesis del positivismo en su contexto científico. Cuadernos críticos de Geografía Humana. Universidad de Barcelona, año IV. n. 19, enero 1979.

NIETZSCHE, F. La gaya Ciencia. Alemania: [s/d], 1882. Versión digital disponible en: http://www.edu.mec.gub.uy/biblioteca_digital/libros/N/Nietzsche\%20-

\%20De\%20La\%20gaya\%20ciencia.pdf

NIETZSCHE, F. Así habló Zaratustra. Alemania: [s/d], 1885. Versión digital en: http://www.enxarxa.com/biblioteca/NIETZSCHE\%20Asi\%20hablo\%20Zaratustra.pdf

POLANYI, M. The tacit dimension. Estados Unidos, Anchor Books, 1967.

POLANYI, M. Science, Faith and Society. The University of Chicago Press. Chicago, 4. ed. 1970.

POPPER, K. La lógica de la investigación científica. Madrid: Editorial Tecnos, 1985. 
ROGERS, E.; SHOEMAKER, F. La comunicación de innovaciones: un enfoque transcultural. Buenos Aires: Centro Regional de Ayuda Técnica, 1974.

REALE, G.; ANTISERI, D. Historia del Pensamiento filosófico y científico. Tomo tercero, "Del romanticismo hasta hoy", p. 271-319. Barcelona: Editorial Herder, 1988.

SÁNCHEZ, G. El Chamanismo o Schamanismo. [s/d]. Versión digital disponible en: https://encolombia.com/libreria-digital//medicina/pensamiento-magico/chamanismo-oschamanismo/. Acceso: 19 abr. 2018.

SÁNCHEZ DE PUERTA, F. Extensión agraria y desarrollo rural. Sobre la evolución de las teorías y praxis extensionistas. Madrid: Ministerio de Agricultura, Pesca y Alimentación. Secretaría General Técnica, Serie Estudios, n. 123, 1996. p. 69-217.

VÁZQUEZ, F. et al. Las teorías del caos y los sistemas complejos: proyecciones físicas, biológicas, sociales y económicas. Encuentros multidisciplinares, v. 3, n. 7, p. 40-70, 2001. 